\title{
A FOTOQUÍMICA NO BRASIL
}

\author{
Miguel G. Neumann* \\ Instituto de Química de São Carlos, Universidade de São Paulo, CP 780, 13560-970 São Carlos - SP \\ Frank H. Quina \\ Instituto de Química, Universidade de São Paulo, CP 26077, 05513-970 São Paulo - SP
}

PHOTOCHEMISTRY IN BRAZIL. An historical overview is provided of the origins, growth and development of the field of photochemistry in Brazil, with special emphasis on the last three decades.

Keywords: photochemistry; photobiology; photobiochemistry.

\section{CONSIDERAÇÕES INICIAIS: O QUE É A FOTOQUÍMICA?}

Antes de iniciar a nossa análise do crescimento recente da fotoquímica no Brasil, convém examinar brevemente a seguinte questão: o que é a fotoquímica? Desde o seu início, o campo da fotoquímica sempre foi muito mais amplo do que o mero estudo das reações químicas provocadas pela luz. Na realidade, a fotoquímica abrange todos os aspectos da química e física de estados eletronicamente excitados da matéria, desde a sua criação até a sua eventual desativação de volta ao estado fundamental (o estado da matéria não excitado eletronicamente; vide os glossários de termos técnicos da fotoquímica em português ${ }^{1}$ e inglês ${ }^{2}$ da IUPAC). Assim, a fotoquímica engloba estudos:

- de processos de criação do estado excitado, como a excitação por luz ou outra radiação eletromagnética ou ionizante apropriada e a quimiexcitação ou criação de estados excitados por intermédio de reações químicas altamente exotérmicas;

- da dinâmica de relaxação e da redistribuição da energia de excitação entre estados excitados da molécula imediatamente após a excitação;

- dos diversos processos de luminescência através dos quais os estados excitados decaem ao estado fundamental com a emissão de um fóton, tais como a fluorescência e fosforescência, a quimiluminescência, a radioluminescência e a eletroluminescência;

- de todos os processos que desativam o estado excitado através da conversão da sua energia diretamente em calor (conhecidos como transições não radiativas);

- dos mecanismos de transferência da energia de excitação de uma molécula doadora para uma molécula aceptora; e, finalmente,

- das transformações químicas sofridas pelo estado excitado, que resultam na formação de novas espécies químicas. As transformações químicas típicas de estados excitados incluem a fragmentação homo- ou heterolítica da molécula, com a produção de intermediários reativos (radicais livres, carbenos, cátions), rearranjos estruturais da molécula, reações bimoleculares como substituição ou dimerização e reações de óxido-redução.

\footnotetext{
*e-mail: neumann@iqsc.sc.usp.br
}

Ao contrário da química do estado fundamental, a fotoquímica tradicionalmente reluta em subdividir-se em áreas exclusivas de especialização ou restringir-se a um certo tipo de composto ou abordagem teórica ou metodológica, mantendo um elevado grau de interdisciplinaridade. Assim sendo, um congresso típico da área de fotoquímica pode reunir:

- teóricos especializados na química quântica de estados excitados;

- espectroscopistas de diversos tipos (p. ex., vibracional, de luminescência ou de absorção resolvida no tempo) trabalhando em diversas escalas de tempo (com resolução temporal de segundos a fentossegundos);

- químicos interessados na fotorreatividade de compostos orgânicos, inorgânicos e organometálicos;

- fotobiofísicos, fotobiólogos e fotobioquímicos que estudam os processos fotoquímicos que ocorrem em sistemas biológicos em vivo e em vitro (bioluminescência, fotossíntese, visão, quimiexcitação através de reações enzimáticas, produção e reatividade de oxigênio singlete, enzimas ativadas por luz, etc.);

- químicos, bioquímicos e biofísicos que utilizam fenômenos fotofísicos como ferramenta e estados excitados como sondas para investigar sistemas organizados (zeólitos, micelas, membranas, ciclodextrinas, biomoléculas como proteínas etc.);

- pesquisadores interessados nas múltiplas aplicações práticas da fotoquímica. Entre estas aplicações práticas, podemos destacar a fotopolimerização (processos de polimerização iniciados pela absorção da luz, largamente empregados na odontologia e na microeletrônica); a geração de fotoimagens (fotografia, xerografia, holografia e estereolitografia em duas e três dimensões); a fotomedicina ou fototerapia (a prevenção dos danos causados à saúde pela luz e o emprego de estados excitados como agentes terapêuticos, como por exemplo na fototerapia de câncer ou no tratamento de icterícia em recém-nascidos); o desenvolvimento de novos materiais luminescentes, fotocondutores, fotocrômicos (que mudam de cor quando irradiados com luz) ou triboluminescentes (que emitem luz quando tocados ou danificados); a fotoquímica de biomassa; a conversão e armazenamento da energia solar em forma de energia térmica ou química; e a fotoquímica ambiental (a contribuição de reações fotoquímicas à destruição da camada de ozônio e à poluição de atmosferas urbanas via a formação de "smog" fotoquímico, bem como o uso de reações fotocatalíticas para destruir ou "mineralizar" com- 
postos orgânicos presentes em águas ou efluentes industriais).

\section{A “PRÉ-HISTÓRIA" DA FOTOQUÍMICA NO BRASIL}

Mesmo antes do descobrimento do Brasil por Cabral em 1500, os povos indígenas do Brasil haviam percebido que o corante vermelho urucu(m), utilizado na pintura do corpo como enfeite e para repelir insetos voadores, também servia para proteger a pele contra queimaduras do $\mathrm{sol}^{3}$. Conheciam também os insetos bioluminescentes como o pirilampo (uauá em tupi) ${ }^{4}$ e os fenômenos de noctiluca ou fosforescência-do-mar (mbusi'ki em tupi, transformado em buxiqui em português do Brasil) ${ }^{4}$, a bioluminescência emitida por dinoflagelados noctilucídeos quando a água do mar é agitada, e o fogo-fátuo (uma luminescência ou "chama" noturna de origem incerta), interpretado em lendas como o mau espírito dos caminhos (macaiera ou macaxera em tupi $)^{5}$ ou a cobra de fogo (boitatá $)^{4}$.

Os navegantes portugueses e espanhois da época dos descobrimentos conheciam bem o fenômeno de corpo-santo ou de fogo de Santelmo, uma (eletro)luminescência que aparecia, às vezes, na ponta dos mastros e das vergas nos navios em ocasião de tempestades e que era tido como um sinal de proteção divina pelos marinheiros aflitos. Assim, por exemplo, há registros históricos e poéticos do aparecimento do fogo de Santelmo durante a viagem de descobrimento das Índias do Vasco da Gama ${ }^{6}$ :

Vi, claramente visto, o lume vivo

Que a marítima gente tem por santo,

Em tempo de tormento e vento esquivo,

De tempestade escura e triste pranto.

Séculos depois (1832), Charles Darwin, a bordo do navio HMS Beagle, ancorado na foz do Rio da Prata, escreveria para J. S. Henslow que $^{7}$ :

\section{“- Tudo está em chamas. - o céu com relâmpagos. - a água com partículas luminosas, \& até os topos dos mastros terminam numa chama azul. -"}

No final do séc. XVIII e início do séc. XIX, a percepção de fenômenos fotoquímicos e fotofísicos incluía ${ }^{8-10}$ :

- a (quimi)luminescência do elemento fósforo quando entrava em contato com o oxigênio do ar;

- a (quimi)luminescência de algumas coisas comuns, como peixe, carne ou madeira quando apodreciam;

- a luminescência (fosforescência) de sulfetos de cálcio e bário no escuro após exposição à luz do sol;

- as faíscas (triboluminescência) produzidas quando rapadura é martelada com pedaços de mármore;

- a relação entre a luz solar e o crescimento das plantas verdes;

- o papel da luz na coloração da pele humana e no desbotamento ou alteração da cor ou forma de algumas substâncias.

Já nesta época, no Brasil, os viajantes alemães Spix e Martius deixaram o seguinte registro da seiva "fosforescente" (certamente uma quimiluminescência resultante de uma autoxidação) de uma planta encontrada no sertão da Bahia entre Salvador e o Rio São Francisco ${ }^{11}$ :

"A vegetação constava aqui, em parte, de um arbusto sem folhas de eufórbia (Euphorbia phosphorea) $e$, quando os galhos do mesmo foram sacudidos $e$ excitados violentemente pelas caixas de bagagem brotou das feridas um leite branco, que luzia no momento da exsudação. Não quis crer no que via, até que eu mesmo conseguisse por fricção dos cantos agudos dos galhos, provocar o fenômeno. A fosforescência durava, de cada vez, só alguns se- gundos, e era mais viva do que a do pau podre, todavia menos forte do que a auréola luminosa de flores do Dictamnus. Durante esse fenômeno, o termômetro indicava $20^{\circ} \mathrm{R}$ e o eletrômetro de Volta não marcava vestígio algum de eletricidade atmosférica. Examinando diversos talos e galhos, obtive sempre o mesmo resultado; porém, ao cabo de uma hora, quando a temperatura havia baixado a $16^{\circ} R$, não pude mais notar o referido efeito luminoso. Até o Rio São Francisco, encontrei com freqüência a planta, ... mas não tive mais a felicidade de observar a fosforescência, e, por isso, chamo a atenção dos futuros viajantes para o assunto."

A distinção de ser o primeiro pesquisador da área de fotoquímica no Brasil provavelmente pertence ao francês Antoine Hercule Romuald Florence, mais conhecido pelo relato e pelas primorosas obras de arte que realizou durante a Expedição Langsdorff ${ }^{12,13}$. Fascinado pelo desenho e pela aventura, Hercule Florence desembarcou no Rio de Janeiro em 1824, com a idade de vinte anos. No ano seguinte, foi contratado como o segundo desenhista da Expedição Langsdorff e, entre setembro de 1825 e março de 1829, percorreu as províncias de São Paulo, Minas Gerais e Grão Pará junto à Expedição $^{12}$. Em 1830, radicou-se na Vila de São Carlos (atual cidade de Campinas), onde iniciou uma série de trabalhos de pesquisa. Entre estes, deve-se destacar a investigação de um processo de reprodução de imagens que denominou "photographie" 14 . Partindo de informação qualitativa sobre o escurecimento do nitrato de prata na luz, pesquisou a fotossensibilidade de nitrato de prata, cloreto de prata e cloreto de ouro. Já em 1833, isto é, seis anos antes da divulgação da "invenção" da fotografia por Daguerre (o processo do daguerreotipia) na França, Florence havia desenvolvido no Brasil um processo fotográfico que permitia a obtenção de cópias por contato em papel impregnado com uma destas três substâncias fotossensíveis ${ }^{14,15}$. Após a exposição do papel impregnado à luz do sol através de um negativo (um desenho feito sobre vidro), as imagens foram fixadas por lavagem do papel com água (no caso de impregnação com $\mathrm{AgNO}_{3}$ ) ou com uma solução aquosa de amônia (impregnação com $\mathrm{AgCl}$ ou cloreto de ouro). Infelizmente, Florence não divulgou ou patenteou o seu processo na época e a fotografia baseada na fotossensibilidade de haletos de prata acabou sendo associada com o nome de William Henry Fox Talbot, que a redescobriu independentemente na Inglaterra (1834-1839) ${ }^{15}$. Desta forma, o primeiro trabalho de pesquisa em fotoquímica no Brasil, a descoberta isolada da fotografia por Hercule Florence, resgatado após quase $150 \operatorname{anos}^{14,15}$, deixou de ter a repercussão que certamente merecia.

Em janeiro de 1840, poucos meses depois da divulgação da daguerreotipia ao mundo, foi feita uma demonstração desta nova arte no Rio de Janeiro por Louis Compte, abade francês em viagem ao redor do mundo ${ }^{15}$. A daguerreotipia logo ganhou inúmeros adeptos, entre eles o Imperador Dom Pedro II. Durante o séc. XIX, a prática e os conhecimentos de fotografia espalharam-se pelo país ${ }^{15}$. Já no final desse século (1896), por exemplo, o jornal O Estado de São Paulo publicou um procedimento para a impermeabilização de papel $^{16}$.

\section{"Papel impermeável}

Para tornar impermeável o papel, basta sumergil-o em colla forte, a que se tenha ajuntado uma pequena quantidade de ácido acético e bicromato de potássio na proporção de 30 grammas para cada litro. É necessário advertir que o papel deve depois ser seccado á luz, pois secco na escuridão não se tornaria impermeável."

Este procedimento obviamente foi baseado no processo fotográfico conhecido como goma-arábica/dicromato ${ }^{17}$, no qual a exposi- 
ção à luz é feita através de uma máscara, seguida de lavagem em água corrente, para obter uma imagem impermeável no papel.

Nas principais histórias da química no Brasil ${ }^{18,19}$, não se encontra nenhuma referência explícita à existência de pesquisa em fotoquímica antes de 1970. No entanto, merecem menção os nomes de dois pesquisadores alemães que realizaram estudos fotoquímicos antes de imigrar para o Brasil ${ }^{19}$. O primeiro destes, o Dr. Hans Stamreich (1902-1969), havia investigado as bases fotoquímicas de processos fotográficos e a validade da lei de Einstein nas reações fotoquímicas (um fóton provoca apenas um processo fotoquímico primário) no Laboratório de Fotoquímica da Escola Politécnica de Berlim-Charlottenburg. Chegando ao Brasil em 1940, o Prof. Stamreich fundou o Laboratório de Espectroscopia Molecular do Depto. de Física da Faculdade de Filosofia da USP (posteriormente transferido para o IQ-USP, onde permanece em pleno funcionamento), no qual desenvolveu trabalho reconhecido internacionalmente no campo da espectroscopia Raman. O segundo pesquisador, o Dr. Hans Zocher (1893-1969), havia desenvolvido trabalhos sobre a química de colóides e mesofases, quimiluminescência e fotoquímica como Professor de Físico-Química das Universidades de Berlim e Praga. Chegando ao Rio de Janeiro em 1946, trabalhou ao lado de Fritz Feigl no Laboratório de Produção Mineral do Ministério da Agricultura, onde se concentrou nos estudos da química de colóides e das propriedades ópticas de cristais. Trabalhando afastado dos centros universitários, Zocher não chegou a formar escola.

\section{A FOTOQUÍMICA NO BRASIL NAS ÚLTIMAS TRÊS DÉCADAS}

O surgimento de linhas formais de pesquisa em fotoquímica nas universidades brasileiras coincide com a escolha da fotoquímica como uma das áreas apoiadas pelo Programa CNPq-NAS ${ }^{19,20}$, um programa experimental de cooperação internacional entre o CNPq e a Academia de Ciências dos Estados Unidos, que funcionou no período de 1969-1977. Através de colaborações entre o Prof. Dr. George S. Hammond do CALTECH e os Profs. Drs. Vicente G. Toscano e W. Bruce Kover, foram estabelecidos Laboratórios de Fotoquímica Orgânica no IQ-USP e no IQ-UFRJ, respectivamente. No período de 1971-1977, ambos laboratórios contaram com a participação de vários recém-doutores americanos (David Mog e David Nicodem no Rio de Janeiro; Richard G. Weiss e Frank Quina em São Paulo) nas tarefas de montagem dos laboratórios e treinamento de pessoal, bem como no desenvolvimento das primeiras atividades de ensino e de orientação de alunos de pós-graduação, especialmente na área de fotoquímica orgânica. Ambos laboratórios permanecem em atividade e tiveram efeito multiplicador, direta e indiretamente, na disseminação da pesquisa em fotoquímica no país.

Uma outra cooperação inserida no Programa CNPq-NAS ${ }^{20}$ foi na área de química de coordenação, envolvendo os Profs. Drs. Henry Taube da Stanford University, Harry B. Gray do CALTECH e Ernesto Giesbrecht do IQ-USP. Embora não visasse explicitamente a implantação de estudos na área de fotoquímica inorgânica, esta colaboração disseminou uma apreciação da fotoquímica e da fotofísica como ramos importantes da química de coordenação, imprescindíveis para a compreensão de processos de transferência de elétrons. $O$ fato que boa parte dos grupos de fotoquímica inorgânica do Estado de São Paulo tiveram alguma associação com os participantes da colaboração ou com o laboratório que foi estabelecido no IQ-USP realça a sua importância.

Em retrospecto, o Programa CNPq-NAS surgiu num momento bastante propício para a área de fotoquímica. O início do Programa coincidiu com o ínicio das atividades de pós-graduação formal no país ${ }^{20}$. Durante o período de implantação e consolidação dos dois laboratórios estabelecidos pelo Programa (1971-1980), surgiram vários outros núcleos de pesquisa (como por exemplo, o Grupo de Fotoquímica do IFQSC, USP-São Carlos) que mais tarde teriam um papel importante no desenvolvimento do campo. Também começaram a aparecer diversos grupos independentes em áreas complementares da fotoquímica, tais como a fotoquímica induzida por lasers (C.T. Lin na UNICAMP), fotofísica (I. Brinn na UFRJ), fotoquímica de compostos de coordenação (M. A. De Paoli na UNICAMP) e a fotobioquímica (G. Cilento e R. Meneghini no IQ-USP; R. Tyrell e L. Caldas no RJ). Esta proliferação de grupos, em geral liderados por pesquisadores com boa experiência no exterior, contribuiu para a rápida formação de uma massa crítica de competência em fotoquímica.

Os anos entre 1980-1990 representam um período de crescimento gradativo, com a consolidação de novos grupos e a formação das primeiras escolas, principalmente no interior paulista (UNICAMP, USP-São Carlos, USP-Ribeirão Preto, UNESP-São José do Rio Preto, UNESP-Araraquara) e no Rio de Janeiro (UFRRJ). Houve um aumento progressivo da disponibilidade de aparelhagem especializada essencial para estudos fotoquímicos e fotofísicos. Em 1990, o equipamento mais habitual para as pesquisas em fotoquímica era o fluorímetro, com o qual se contava em boa parte dessas instituições. Também estavam disponíveis alguns poucos sistemas para a determinação de rendimentos quânticos de fotorreação, um sistema analógico para a determinação de tempos de vida de fluorescência e um aparelho de fotólise de relâmpago convencional ("flash photolysis") com resolução de microssegundos.

A partir da realização do Primeiro Encontro Informal Brasileiro de Fotoquímica e Fotobiologia (EIBhv) em Campinas em outubro de 1981, sugiu a consciência da existência de uma comunidade fotoquímica nacional significativa, o que foi reafirmado nos Encontros seguintes em São Paulo (1983) e Rio de Janeiro (1986). A realização do Primeiro Encontro Latino-Americano de Fotoquímica e Fotobiologia (ELAFOT) em 1982 em Santiago, Chile, iniciou a integração da comunidade brasileira com os colegas de Chile e Argentina. Esta integração tornou-se definitiva com a fusão do Segundo ELAFOT com o Quarto EIBhv no Encontro realizado em 1988 em São Carlos. Este também foi o primeiro encontro do gênero com participação mais expressiva de pesquisadores europeus e norteamericanos.

Comparado com os quinze anos anteriores, o período de 19912001 foi uma fase de crescimento muito mais acelerada da área. Este crescimento está evidenciado na Figura 1 pelo brusco aumento na taxa anual de publicação internacional na área de fotoquímica após 1990 e, na Figura 2, pelo aumento do número de publicações em revistas nacionais (coincidindo com a publicação em 1993 de um número especial da Química Nova dedicado ao Prof. Cilento ${ }^{21} \mathrm{e}$, em 1995, de um número especial do Journal of the Brazilian Chemical Society sobre fotoquímica ${ }^{22}$ ). Convém mencionar que, nesta compilação, desconsideramos trabalhos que tratam apenas da aplicação de técnicas rotineiras, tais como deteção fluorimétrica em HPLC ou o emprego de indicadores ou marcadores fluorimétricos em sistemas bioquímicos ou biológicos. A partir de 1992, a fotoquímica passou a ter uma Seção própria dentro da SBQ.

A integração com a comunidade latino-americana vem se mantendo através de sucessivos ELAFOTs em Mar del Plata (Argentina, 1991), Valparaiso (Chile, 1994), Los Cocos (Argentina, 1997), Teresópolis (Brasil, 1999) e Viña del Mar (Chile, novembro de 2002). $\mathrm{O}$ reconhecimento internacional da existência de uma massa crítica em fotoquímica na América Latina veio com a realização da reunião anual da Sociedade Inter-Americana de Fotoquímica (IAPS) em Foz do Iguaçu em 1996. Com base no sucesso desta reunião, a IAPS criou um fundo especial para estimular a participação de jovens 


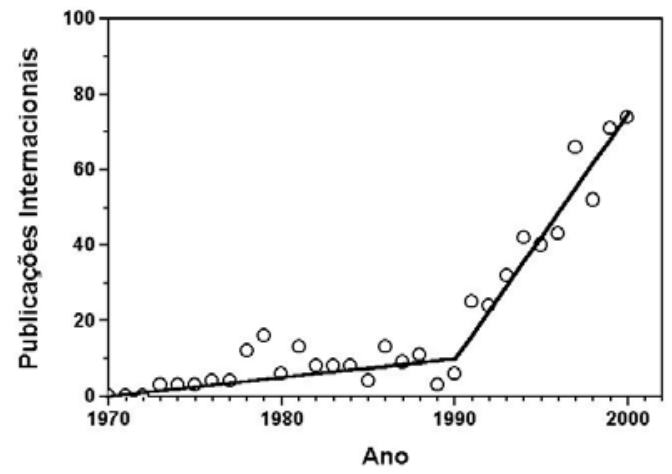

Figura 1. Evolução do número de publicações de pesquisadores nas diversas especialidades de fotoquímica no Brasil em revistas internacionais. Foram compilados artigos de fotoquímica indexados na base de dados "Web of Science" do Instituto for Scientific Information que incluiam Brasil no endereço de um dos autores. As retas correspondem a um acréscimo cumulativo da taxa de publicação de 0,5 artigos por ano entre 1970-1990 e de 6,5 artigos por ano após 1990. Até o início de outubro, contabilizou-se 56 artigos no ano de 2001

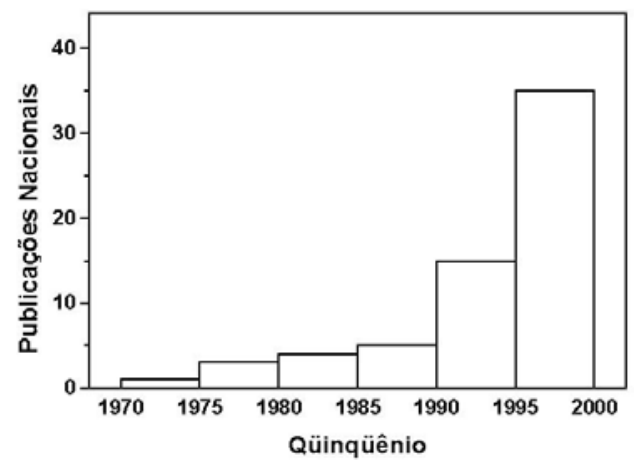

Figura 2. Evolução do número de publicações de pesquisadores brasileiros na área de fotoquímica em revistas nacionais. A compilação, feita inicialmente no "Web of Science", foi complementada através de uma busca manual na Química Nova e no Journal of the Brazilian Chemical Society fotoquímicos da América Latina nas reuniões da IAPS ("Cilento Award Fund"), reservou um lugar permanente para um representante da América Latina no Conselho Executivo da Sociedade e assumiu o compromisso de realizar qüinqüienalmente a sua reunião anual na América Latina (a reunião de 2001 da IAPS foi realizada em Ascochinga, Argentina). Deve-se mencionar ainda a recente realização de dois congressos no Brasil envolvendo aspectos biológicos e ambientais da fotoquímica: (i) 'Singlet Molecular Oxygen, Chemical, Biological and Medical Aspects', realizado em Caraguatatuba, SP, em setembro de 1998; (ii) o 'Primeiro Encontro sobre Aplicações Ambientais de Processos Oxidativos Avançados' em Águas de São Pedro, SP, em outubro de 2001.

Um elemento essencial no crescimento observado nesta última década tem sido a disponibilidade de recursos para a modernização dos equipamentos necessários para o desenvolvimento de trabalhos competitivos no campo. O atual conjunto de equipamentos disponíveis no país para estudos fotoquímicos e fotofísicos inclui inúmeros fluorímetros dispersos pelo país. Nas universidades do RJ e SP, há pelo menos seis aparelhos modernos para a determinação de tempos de vida de fluorescência (cinco baseados no princípio de contagem de fótons únicos, "single photon counting", dois dos quais com resolução de picossegundos, e um espectrômetro de modulação de fase), pelo menos cinco sistemas de fotólise por pulso de laser (Nd-YAG) com resolução de alguns nanossegundos e dois sistemas para a detecção direta do oxigênio singlete.

Embora necessariamente qualitativa, uma classificação por assunto dos trabalhos de pesquisa publicados ou apresentados em reuniões internacionais nos últimos três anos demonstra um excelente desenvolvimento da fotoquímica no Brasil, com uma abrangência impressionante de interesses e competências. Assim, sem detalhá-los, encontramos grupos fortemente engajados em uma gama de estudos fotoquímicos em diversas instituições, como pode ser visto na Tabela 1.

Desta relação de áreas de atuação, fica patente a maturidade alcançada pelo campo de fotoquímica no Brasil nos últimos 25 anos. Atualmente, a aparelhagem fotofísica especializada disponível nos principais centros do país permite a realização de estudos de processos fotofísicos e fotoquímicos em condições de igualdade com os melhores centros internacionais. Ao mesmo tempo, nota-se um crescimento nítido do número de grupos que utilizam a metodologia

Tabela 1. Áreas Atuais de Pesquisa em Fotoquímica no Brasil

\begin{tabular}{ll}
\hline ÁREA & CENTROS \\
\hline Fotoquímica orgânica & USP-SP, UFRJ, UFRRJ \\
Fotofísica em meios homogêneos & USP-SP, USP-SC, UFRJ, UFF, UFBA \\
Fotoquímica inorgânica & USP-SP, USP-SC, USP-RP \\
Luminescência de complexos inorgânicos & USP-SP, USP-RP, UNESP-Araraquara, UFPE \\
Sondas fotofísicas em sistemas organizados & USP-SP, USP-SC, USP-RP, UFRRJ, UNICAMP, UNESP-SJRP, \\
Sondas fotofísicas em sistemas poliméricos & UFRGS, UFSC \\
Sondas fotofísicas associadas com macromoléculas biológicas & UNICAMP, USP-SC, USP-SP, UFRGS \\
Fotoquímica de polímeros e processos de fotopolímerização & UFRJ, USP-SC, UNIFESP, USP-SC, USP-SP \\
Fotofísica de novos materiais & USP-SC, USP-SP \\
Química quântica aplicada a estados excitados e teoria fotofísica & UNICAMP, USP-SC, USP-SP \\
Fototerapia & USP-SP, UNICAMP, USP-RP, UFPE, UFMG \\
Quimiluminescência e Bioluminescência & USP-RP, UFU, UNICAMP \\
Fotoquímica ambiental & USP-SP \\
Fotoquímica de biomassa & UNICAMP, UFRJ, USP-SP, UFBA \\
Conversão e armazenamento de energia solar & UNICAMP, UFU, UFPR \\
\hline
\end{tabular}


fotofísica como uma ferramenta nas mais diversas áreas da química e bioquímica. Assim, enquanto a Plataforma Lattes registra apenas 24 grupos cujo alvo principal declarado são os estudos na área própria de fotoquímica, existem mais de 150 linhas de pesquisa que mencionam fotoquímica nas palavras-chave ou como parte das pesquisas que estão sendo realizadas.

\section{REFLEXÕES FINAIS}

Para concluir a presente análise, cabe uma breve reflexão sobre as perspectivas da fotoquímica no Brasil nas próximas décadas. De um modo geral, a tendência da fotoquímica no país vem acompanhando a evolução do campo no cenário internacional. De modo geral, a tendência é prestigiar cada vez mais as aplicações práticas da fotoquímica e o emprego da fotofísica como uma ferramenta para solucionar problemas em outras áreas da química e bioquímica, deixando de lado os estudos mais clássicos de mecanismos de reações fotoquímicas ou da fotofísica de classes específicas de compostos. Deve continuar forte, portanto, o interesse no emprego de técnicas fotofísicas em estudos básicos de dinâmica, mobilidade, microestrutura e reatividade em sistemas organizados e macromoleculares e para gerar e estudar intermediários reativos. Em algumas áreas extremamente promissoras da fotoquímica, como a fototerapia e a química quântica ( $a b$ initio) de estados excitados, a pesquisa ainda é incipiente, mas deve atingir um nível razoável de consolidação a médio prazo. Aplicações práticas da fotoquímica em áreas como a química de materiais, a química ambiental e o aproveitamento racional da energia da luz solar devem ser cada vez mais importantes, principalmente se conseguirem envolver uma faixa maior da comunidade fotoquímica neste esforço.

Embora saudável para a pesquisa básica, a interdisciplinaridade da fotoquímica e a ênfase nas aplicações de metodologia fotoquímica em outras áreas do conhecimento acaba criando uma certa crise de identidade para a fotoquímica. Cada vez mais, os fotoquímicos que se engajam na aplicação de metodologia fotofísica à solução de problemas de outras áreas, tais como a química coloidal, a química macromolecular ou a química de materiais, acabam se identificando com as comunidades dessas áreas. Por outro lado, pesquisadores destas outras comunidades incorporam a metodologia fotofísica no seu arsenal de ferramentas rotineiras, sem se integrar à comunidade fotoquímica. Portanto, além dos desafios da pesquisa, a fotoquímica terá que encontrar meios de sobreviver como um campo específico do conhecimento. Cientes destas tendências, a Royal Chemical Society, a European Society for Photobiology (ESP) e a European Photochemistry Association (EPA) acabam de fornecer um importante apoio ao campo, lançando (janeiro de 2002) a revista especializada Photochemical and Photobiological Sciences ${ }^{23}$.

\section{REFERÊNCIAS}

1. Neumann, M. G.; Burrows, H. D.; Quim. Nova 1993, 16, 71.

2. Verhoeven, J. W.; Pure Appl. Chem. 1996, 68, 2223.

3. Pereira, M. N.; Moronguêtá: Um Decameron Indígena, $2^{\mathrm{a}}$ ed., Civilização Brasileira: Rio de Janeiro, e INL-MEC: Brasília, 1980, vol. 1, p. 152

4. Ferreira, A. B. H.; Novo Dicionário da Língua Portuguesa, 2a ed., Ed. Nova Fronteira: Rio de Janeiro, 1986.
5. Silveira Bueno, F.; Vocabulário Tupi-Guarani Português, 6 $6^{\mathrm{a}}$ ed., Éfeta Ed.: São Paulo, 1998.

6. Camões, L.; Os Lusíadas, canto V, estrofe 18.

7. Barlow, N.; Darwin and Henslow, The Growth of an Idea: Letters 18311860, Univ. of California Press: Berkeley, 1969, p. 59.

8. Seabra, V. C.; Elementos de Chimica, Facsímile da edição de 1788 (Parte I) e 1780 (Parte II), Depto. de Química, Universidade de Coimbra: Portugal, 1985 .

9. Olmstead, D.; Outlines of the Lectures on Chemistry, Mineralogy, \& Geology Delivered at the University of North Carolina, J. Gales: Raleigh, NC, 1819 , p. 6

10. Roth, H. D.; Angew. Chem., Int. Ed. 1989, $28,1193$.

11. Martius, C. F. P.; Spix, J. B.; Viagem pelo Brasil, 1817-1820, Ed. Itatiaia: Belo Horizonte e EDUSP: São Paulo, 1981, vol. 2, p. 207.

12. Florence, H.; Viagem Fluvial do Tietê ao Amazonas de 1825 a 1829, Ed. Cultrix/EDUSP: São Paulo, 1977.

13. Costa, M. F. G.; Diener, P.; Strauss, D.; O Brasil de Hoje no Espelho do Século XIX: Artistas Alemães e Brasileiros Refazem a Expedição Langsdorff, Ed. Estação Liberdade: São Paulo, 1995.

14. Kossoy, B.; Hercule Florence, 1833: A Descoberta Isolada da Fotografia no Brasil, 2 a ed., Livraria Duas Cidades: São Paulo, 1980.

15. Kossoy, B.; Origens e Expansão da Fotografia no Brasil, Século XIX, MEC/FUNARTE: Brasília, 1980.

16. O Estado de São Paulo, 15 de julho de 1996, Seção "Há Um Século".

17. Monforte, L. M.; Fotografia Pensante, Ed. SENAC: São Paulo, 1997, p. 117-131.

18. Rheinbolt, H. Em As Ciências no Brasil; Azevedo, F., ed.; Ed. Melhoramentos: São Paulo, 1955, vol. II, p. 9-89.

19. Matias, S.; Cem anos de química no Brasil, Coleção da Revista de História LXIII São Paulo, Brasil, 1975; Suplemento do Centenário, No. 6, O Estado de São Paulo de 8 de fevereiro de 1975.

20. Riveros, J. M. Em Paschoal Senise, Uma Carreira Dedicada à Educação; Colli, W., ed.; Paralelo 15 Ed. e CAPES, MEC: Brasília, 2001, p. 73-76.

21.Tedesco, A. C.; Nogueira, L. C.; Bonilha, J. B. S.; Alonso, E. O.; Quina, F. H.; Quim. Nova 1993, 16, 275.

22. J. Braz. Chem Soc. 1995, 6, 111-220. Special Issue on Photochemistry and Photobiology.

23. http://www.rsc.org/pps

\section{APÊNDICE}

\section{DIVISÃO DE FOTOQUÍMICA}

\section{Número de filiados: 74 (atual)}

2000-2002

DIRETOR: Marcio Jose Tiera

VICE-DIRETOR: Ira Mark Brinn

1998-2000

DIRETOR

VICE-DIRETOR:

Antonio Eduardo da Hora Machado

Ira Mark Brinn

1996-1998

DIRETOR:

VICE-DIRETOR:

David Ernest Nicodem

Frank Herbert Quina

1994-1996

DIRETOR:

VICE-DIRETOR:

Miguel G. Neumann

TESOUREIRO
João Batista Sargi Bonilha

José Carlos Netto Ferreira 